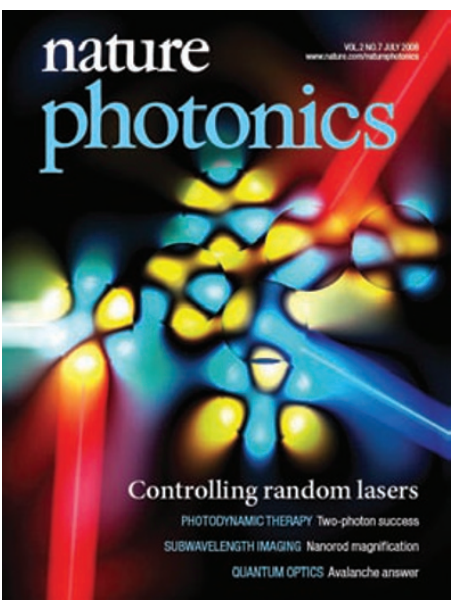

Cover story

Vol.2 No.7 July 2008

Random lasers, coherent light sources that use an assembly of light-scattering particles rather than cavity mirrors to achieve the necessary optical feedback for lasing, are a topic of emerging interest in photonics. However, until now, predicting and controlling the behaviour of random lasers, in particular the emission wavelength, has been a difficult challenge. Now Stefano Gottardo and co-workers have demonstrated that it is actually possible to construct a random laser with a pre-determined and controllable wavelength. Their answer lies with the use of Mie resonance scattering from 'photonic glass' based on an assembly of polystyrene microspheres mixed with a laser dye. The wavelength of the Mie resonance and hence lasing can be controlled by the diameter and refractive index of the spheres. [Letter p429; News \& Views p397; Interview p446]

\section{QUANTUM COUNTING}

Determining the exact number of photons in a very weak light pulse is important for performing reliable experiments in quantum optics and computing. Unfortunately, it is a very difficult task, and so far such 'photon-number-resolving detectors' have been complex and suffered from issues such as a poor efficiency, the need for cooling to cryogenic temperatures or a slow response time. Now, Andrew Shields and co-workers from Toshiba Research Europe, have demonstrated a design of a photonnumber detector that is simple, efficient and cost-effective.

Shields and his co-workers report a specially adapted avalanche-photodiode detector (APD) and show that it is able to discriminate between weak laser pulses containing different numbers of photons - such as $0,1,2$ or 3 . Avalanche photodiodes are routinely used to detect weak light signals. They rely on an avalanche multiplication effect whereby a single photon generates a large number of electrons and thus an electrical current that is easy to detect. Usually this avalanche effect masks the actual number of photons received and makes determining the exact number received impossible. The Toshiba researchers solved this problem by implementing an electronic circuit that measures the avalanche currents at a much earlier stage in their development, allowing the number of photons striking the detector to be determined.

[Letter p425; News \& Views p392]

\section{COLOURFUL APPROACH}

The development of practical techniques for performing imaging with subwavelength resolution is of great interest to many researchers in optics, especially those involved in biological imaging of cells. In this issue, Satoshi Kawata and coworkers report an approach, supported by theoretical simulations, for subwavelength imaging that is compatible with colour transmission and operates over far-field distances. Rather than using metamaterials, an approach that has been the topic of intense research recently, Kawata's team propose the use of stacked silver nanorods and plasmonics. The idea is that the evanescent light field from the object being imaged excites plasmons in the nanorods, and these plasmons travel along the stack before being converted back to an optical signal to be imaged by a conventional detector. Calculations indicate that the image could be transferred over micrometre-scale distances and that image magnification is possible by tapering the stack of nanorods to make the stack diameter larger at the user end.

[Article p438; News \& Views p387]

\section{PRECISE PHOTODYNAMIC THERAPY}

Photodynamic therapy (PDT), which uses a light-activated drug to kill cells, is a popular optical technique for treating diseases such as cancer. But researchers would like the technique to be more precise and to be able to penetrate deeper into biological tissue. The use of photosensitizer drugs that are activated by two-photon excitation (TPE)

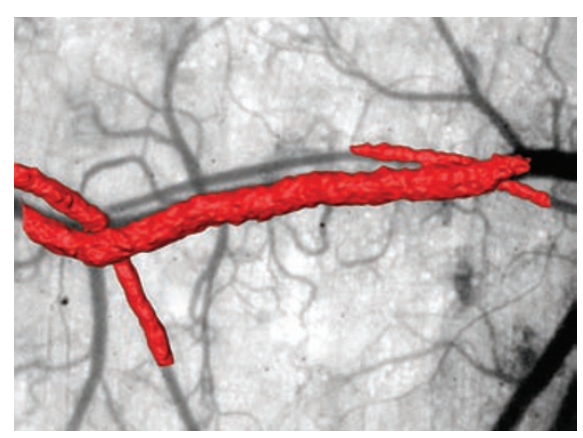

Photodynamic therapy based on two-photon excitation is able to selectively close single blood vessels. could be the answer, as it allows the use of infrared rather than visible light, which means less absorption and scatter in tissue. In addition, excitation is confined to the focal spot of the laser beam. Unfortunately, so far drugs have featured very low two-photon absorption cross-sections thus hindering the development of TPE-PDT. In this issue, Harry Anderson and colleagues describe a new family of two-photon photosensitizer drugs that have far stronger light-absorption characteristics (two magnitudes stronger than conventional clinical photosensitizers) and report TPE-PDT-induced selective closure of single blood vessels in vivo in a mouse. The experiments represent the first demonstration of TPE-PDT in a mammal.

[Letter p420; News \& Views p394]

\section{EFFICIENT COMMUNICATION}

An important issue for the IT and communications industry is finding and developing more energy-efficient ways of transmitting data bits. As bandwidth demands and data capacity continue to spiral upwards, it is vital that data can be passed around networks and circuit boards in an energy-efficient manner. With this aim in mind, Jifeng Liu and co-workers have developed a waveguide-integrated GeSi on silicon optical modulator that consumes just $50 \mathrm{fJ}$ of electrical energy per data bit. The modulator is based on the electro-absorption effect (applying a voltage shifts the material's bandgap between absorption and transparency, thus achieving optical modulation). It's small size $\left(30 \mu \mathrm{m}^{2}\right.$ active area) and compatibility with CMOS techniques for fabricating electronics make the approach promising for future hybrid silicon chips that integrate photonics and electronics. The key issue now is to improve the device's bandwidth of $1.2 \mathrm{GHz}$ to higher speeds, a task that the researchers are confident is possible.

[Letter p433; News \& Views p389] 